\title{
The Sea Urchin sns Insulator Blocks CMV Enhancer following Integration in Human Cells
}

\author{
Paola Di Simone, Aldo Di Leonardo, Giorgia Costanzo, Raffaella Melfi, and Giovanni Spinelli ${ }^{1}$ \\ Department of Cell and Devel opmental Biology, "A.Monroy" University of Palermo, \\ viale delle Scienze, 90128 Palermo, Italy
}

Insulators are a new class of genetic elements that attenuate enhancer function directionally. Previously, we characterized in sea urchin a 265-bp-long insulator, termed sns. To test insulator activity following stable integration in human cells, we placed sns between the CMV enhancer and a tk promoter upstream of a GFP transgene of plasmid or retroviral vectors. In contrast to controls, cells transfected or transduced with insulated constructs displayed a barely detectable fluorescence. Southern blot and PCR ruled out vector rearrangement following integration into host DNA; RNase protection confirmed the enhancer blocking activity. Finally, we demonstrate that two cis-acting sequences, previously characterized in sea urchin, are also specific binding sites for human proteins. We conclude that sns interferes with enhancer promoter interaction also in a human chromatin context. The relatively small size, evolutionary conservation and apparent lack of enhancer specificity might result useful in gene transfer experiments in human cells. ๑ 2001 Academic Press

Increasing evidence indicates that insulators are regulatory elements that separate functional domains in eukaryotic chromosomes. Insulators are commonly referred as enhancer blocking elements, in that they buffer promoters from the action of enhancers or silencers only when placed between them (reviewed in (1-3). In addition, some insulators confer position independent expression of transgenes when placed in flanking locations (4). The earliest to be described, and the best characterised are the Drosophila scs and Scs', and the tandemly repeated binding sites for suppressor of hairy wing $[\mathrm{su}(\mathrm{Hw})]$ protein in the gypsy retrotransposon (5-9). Another well characterized insulator is the 1.2-Kb cHS4 of the chicken globin LCR locus (10).

\footnotetext{
${ }^{1}$ To whom correspondence should be addressed at Dipartimento di Biologia Cellulare e dello Sviluppo, Parco d'Orleans II, 90128 Palermo, Italy. Fax: +39091/6577430. E-mail: spinelli@unipa.it.
}

cHS4 contains a binding site for the CTCF transcription factor, which in tandem array displays significant enhancer blocking activity in both transient and stable transfection assays $(11,12)$. CTCF also binds to several other vertebrate insulators, including the unmethylated ICR (imprinting-control region) that exhibits enhancer blocking activity and controls imprinted expression of the Igf2 gene $(13,14)$.

Because insulators can attenuate enhancers and can in certain circumstances counteract silencing effects on gene expression by repressive chromatin (15), they could be extremely useful tools in the design of expression vectors for gene delivery experiments. I ndeed, cHS4 reduces position effects in transgenic animals (16), increases the fraction of transduced cells expressing integrated retroviral vectors $(17,18)$, and improves expression from an inducible promoter in episomal adenoviral vectors (19). To have wide practical application in gene transfer by viral vectors, an insulator should be small and lack enhancer or cell type specificity. In this regard, it is of great interest to test functionally the ability of insulators to shield promoters from different enhancers and in different cell types.

We have previously described the identification in sea urchin of a DNA element with the characteristics of an insulator. This element, designated sns (silencing nucleoprotein structure), is 265 bp long and is localized at the $3^{\prime}$ end of the H2A gene of the early histone repeating unit of the sea urchin Paracentrotus lividus. The sns itself has a transcriptionally neutral role, but displays the ability to block the interaction of the histone H2A enhancer with the Herpes simplex tk promoter. Blocking activity is observed only when sns is placed between the enhancer and the promoter, in either orientation (20). The sns can interfere with enhancer promoter interaction at a great distance from the promoter, and does so in a directional manner (20, 21). Remarkably, sns also blocked the function of the SV40 enhancer in transiently transfected human cells, suggesting the absence of enhancer specificity and the evolutionary conservation of insulator activity (20). 
As a first step toward using sns as an insulator in human cells, we stably transfected human cells with plasmid vectors in which sns was placed between the CMV enhancer and a tk promoter driving the expression of a green fluorescent protein (GFP) transgene. We present evidence for a drastically reduced expression of the reporter gene in several cell lines strongly suggesting that sns is able to work as an insulator of enhancer activity within the context of human chromatin.

\section{MATERIALS AND METHODS}

Plasmids. To generate plasmid A (Fig. 1), the 106 bp tk basal promoter fragment was isolated from a tk-CAT vector (22), by Bglll and Sall double digestion and cloned in to the BamHI and Sall sites, between the CMV region and the EGFP gene, of the pEGFP-N1 vector (CLONTECH). To create plasmid $B$, the 265 bp sea urchin sns insulator, isolated from a histone H2A gene subclone (20), was inserted in the Sall site of plasmid A. In plasmid C, a tandem array, $230 \mathrm{bp}$ long, of an unrelated oligonucleotide substituted the sns insulator. In plasmid D, the sns insulator was inserted in the Asel filled site of plasmid A. The retroviral plasmids $E$ and $F$ were constructed by cloning, respectively, the tk-EGFP cassette or the snstk-EGFP fragment isolated from plasmids A and B in the polylinker region of the PSLX vector, a gift of Dr. Shu, UCSD, San Diego, CA.

Transfection and transduction of human cells. Human U2-OS and $\mathrm{H} 1299$ tumor cells were cultured (at $37^{\circ} \mathrm{C}$ in $5 \% \mathrm{CO}_{2}$ ) in DMEM supplemented with $10 \%$ FBS (Euroclone). pE GFP DNA derived plasmids were transfected into $4 \times 10^{6}$ exponentially growing U2-OS cells by calcium phosphate and selected with $400 \mu \mathrm{g} / \mathrm{ml}$ G418 for 3 weeks. Clones were isolated and expanded for microscopic observation and molecular analysis. The PA317 packaging line was transfected with the pSLX derived retroviral vectors and selected with G418 $400 \mu \mathrm{g} / \mathrm{ml}$ for 2 weeks. Vector containing supernatant was collected from semiconfluent plates and filtered through a $0.22 \mu \mathrm{m}$ filter. U2-OS and H1299 cells were transduced with $3 \mathrm{ml}$ of filtered viral supernatant and $1 \mathrm{ml}$ of complete medium in the presence of polybrene $(4 \mu \mathrm{g} / \mathrm{ml})$ for $3 \mathrm{~h}$. Two infection cycles were performed. Two days after infection, G418 $(400 \mu \mathrm{g} / \mathrm{ml})$ was added and cells were cultured for 2-3 weeks. Microscopic evaluation of EGFP expression in transfected and transduced cells was done using a microscope (ZEISS, Axioskop) equipped for epifluorescence.

Southern blot, PCR analysis, and RNase protection. Ten micrograms of genomic DNA extracted from transfectants were digested with $\mathrm{Xbal}$, run on $0.8 \%$ agarose gel, and blotted onto nylon membrane. The Blot was hybridized with a $\mathrm{P}^{32}$-labelled EGFP probe and after probe stripping, reprobed with a $\mathrm{P}^{32}$-labelled NEO fragment. Signal intensities were quantified by densitometric scanning.

One hundred nanograms of genomic DNA were used to amplify the integrated EGFP transcription unit. Thirty PCR cycles (cycle: denaturation at $95^{\circ} \mathrm{C}$, for $60 \mathrm{~s}$; annealing at $56^{\circ} \mathrm{C}$, for $60 \mathrm{~s}$; extension at $72^{\circ} \mathrm{C}$ for $90 \mathrm{~s}$ ) were performed by using the oligonucleotides $5^{\prime}$ CAATTACGGGGTCATTAGTTC 3' (coordinates 17-38) and 5' ACCACAACTAGAATGCAGTG 3' (coordinates 1604-1625). The PCR products were run on $1 \%$ agarose gel, blotted on nylon membrane and hybridised with $\mathrm{P}^{32}$-labelled EGFP or sns probes. RNase protection on total RNAs was as described (20). The $\mathrm{P}^{32}$-labelled antisense RNAs were transcribed in vitro from EFGP and NEO subclones in pBS.

Electrophoretic mobility shift assay. Nuclear extract from human cells were prepared as standard protocols. Briefly, purified nuclei were resuspended by three strokes in a Dounce homogenizer, in 0.5 $\mathrm{ml}$ (for $10^{10}$ cells) of $20 \mathrm{mM}$ Hepes pH 7.9, $0.55 \mathrm{M} \mathrm{NaCl}, 1.5 \mathrm{mM}$ $\mathrm{MgCl}_{2}, 0.2 \mathrm{M}$ EDTA, $0.5 \mathrm{mM}$ DTT, $0.5 \mathrm{mM} \mathrm{PMSF}, 25 \%$ glycerol, protein extracted for $30 \mathrm{~min}$ on ice and centrifuged at $12000 \mathrm{~g}$ for 30
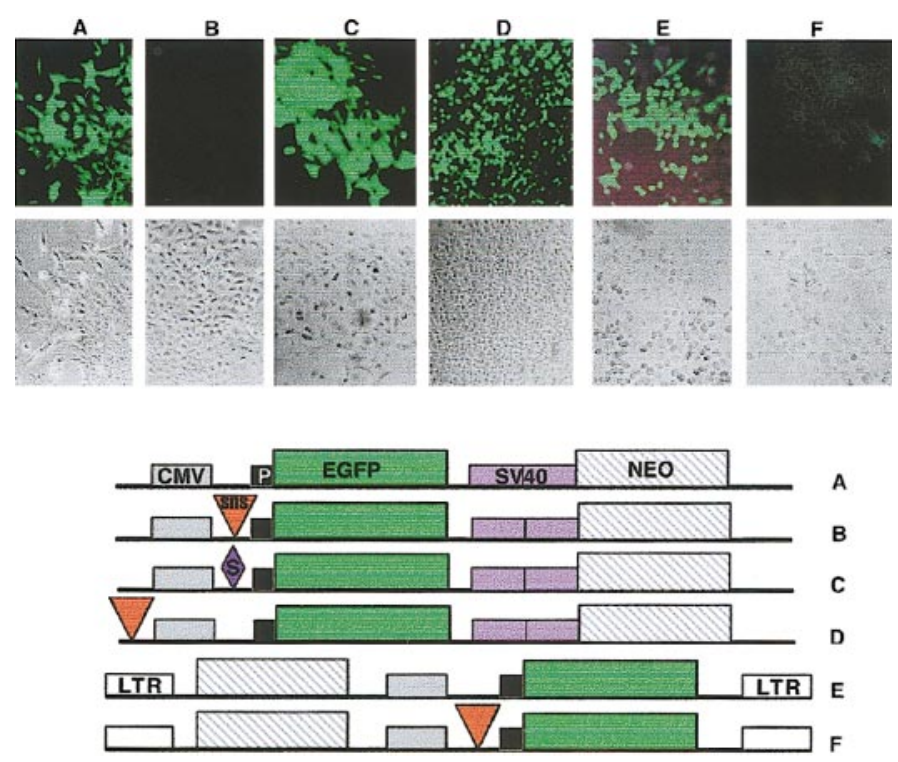

FIG. 1. EGFP transgene expression from insulated and uninsulated constructs stably integrated in human cells. DNA constructs are schematically drawn below the photographs. (A-D) Clones of U2-OS cells transfected with plasmids A, B, C, and D. (E and F) Pools of $\mathrm{H} 1299$ cells infected with retroviral constructs $E$ and $F$. CMV enhancer is blocked by the sns insulator.

min at $4^{\circ} \mathrm{C}$. The supernatant was dialysed in $500 \mathrm{ml}$ of $15 \mathrm{mM}$ Hepes $\mathrm{pH}$ 7.9, $40 \mathrm{mM} \mathrm{KCl}, 0.5 \mathrm{mM}$ DTT, $0.5 \mathrm{mM}$ PMSF , 20\% glycerol, 1 mM EDTA and cleared by centrifugation at $15000 \mathrm{~g}$ for $15 \mathrm{~min}$ at $4^{\circ} \mathrm{C}$. Five micrograms of nuclear extracts were preincubated with $2 \mu \mathrm{g}$ of poly $(\mathrm{dl}-\mathrm{dC}) \cdot(\mathrm{dl}-\mathrm{dC})$ and with or without unlabelled homologous or heterologous oligonucleotides in the amounts described in the legend to Fig. 4 prior the addition of 1 ng labelled probes. The sequence of Box A, Box B, GAGA oligonucleotides were reported in (21). The CTCF oligonucleotide was derived from Bell et al. (11). EMSA analysis of protein DNA complexes were as described (21). Nuclear extracts from sea urchin embryos were prepared as described (22).

\section{RESULTS AND DISCUSSION}

\section{The Sea Urchin sns Element Insulates the CMV Enhancer in Stably Transfected Human Cells}

To assess the insulator function of sea urchin sns in a human chromatin context we tested plasmid and retroviral vectors that are schematically drawn in Fig. 1. The 265 bp sns sequence was placed between the CMV enhancer and the tk promoter upstream of a CDNA encoding the enhanced version of GFP (EGFP). Both types of reporter vectors contain a second expression cassette, the neomycin gene (neo), driven by the SV40 enhancer or LTR enhancer, as a selectable marker. Two further plasmids were constructed. In one plasmid, an unrelated spacer fragment of similar length to sns, was interposed between the enhancer and the promoter. In addition, to distinguish between directional enhancer blocking and bidirectional silencing, sns was also cloned upstream the CMV enhancer. We first transfected the plasmid constructs (Figs. 1A- 

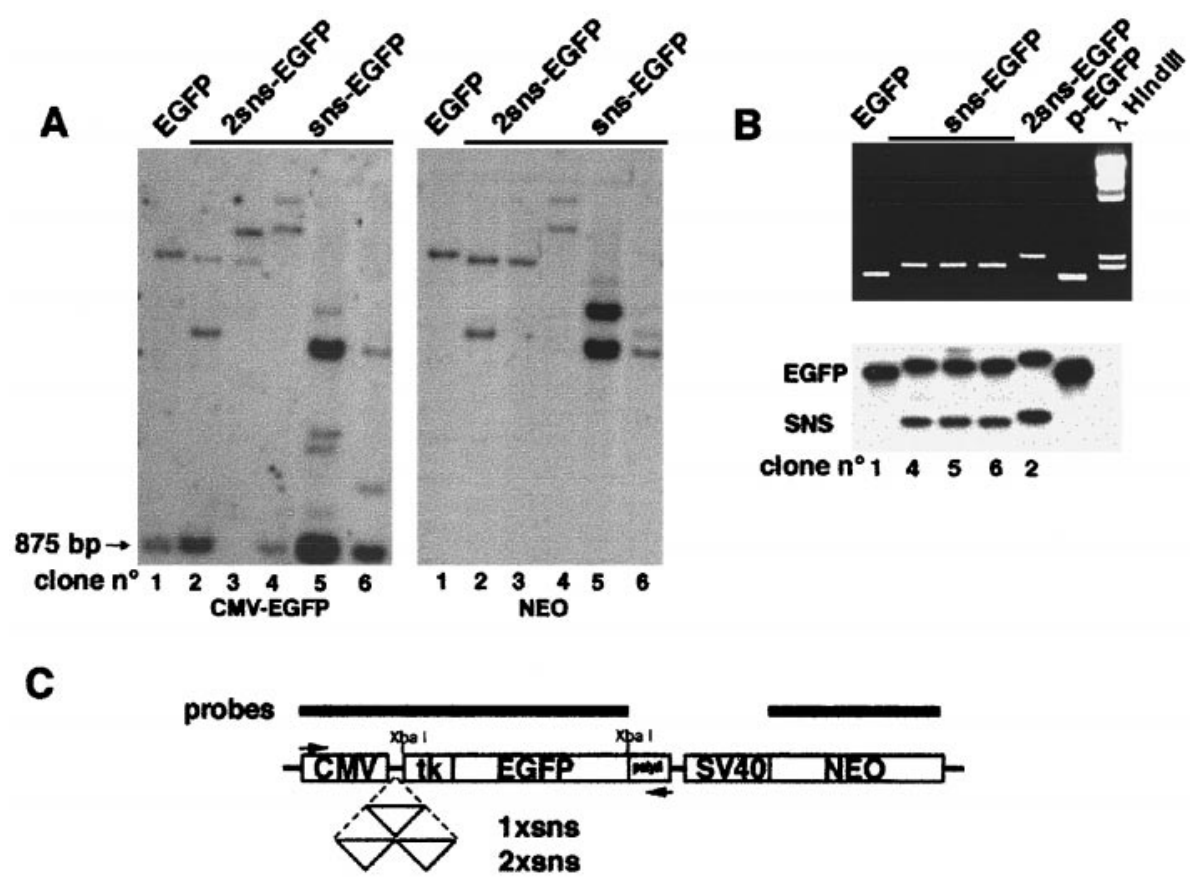

FIG. 2. Molecular analysis of the integrated transgenes. (A) Southern blot of Xbal digested genomic DNA extracted from one uninsulated and five insulated U2-OS transfectants. The same filter was hybridised with a CMV-EGFP probe (left) and, after probe stripping, with the NEO probe (right). Clone 1, transfected with the pEGFP control; clones 2 and 3, transfected with insulated pEGFP plasmid containing two tandem copies of sns; clones 4-6, transfected with EGFP construct containing one copy of sns. (B) PCR analysis of integrants. The EGFP transcription unit was amplified from DNA from all but clone 3, cellular clones shown in A using oligonucleotides located respectively at the $5^{\prime}$ end of CMV and at 3' end of the second polyadenylation signal. PCR products were hybridized with the EGFP and sns probes, respectively. (C) Map of the uninsulated and insulated transgenes. One and two copies of sns are indicated. Probes are overlined. Arrows point to forward and reverse primers, respectively.

1D) into the U2-OS cells. Following three weeks of selection, a number of resistant colonies were analyzed by phase contrast and fluorescence microscopy to monitor EGFP expression. The great majority of clones transfected with the EGFP control plasmid displayed a bright green fluorescence (Fig. 1A). By contrast, GFP gene expression was barely detectable in cells transfected with the insulated plasmid containing sns in forward (Fig. 1B) and inverted orientation (not shown). Two lines of evidence indicate that the drastic reduction in reporter gene expression observed with the insulated construct B can only be explained by shielding of the promoter from the enhancer, rather than by the increased distance between enhancer and promoter or by promoter silencing. First, cells transfected with the control spacer construct exhibited a bright fluorescent phenotype (Fig. 1C). Second, the enhancer blocking function was position-dependent, in that the sns fragment placed upstream the enhancer did not affect transcription of the GFP gene (Fig. 1D).

I dentical results were obtained with retroviral constructs (Fig. 1E and 1F). Pools of transduced H1299 or U2-OS cells grown under selection, were observed under a fluorescence microscope. Again, most cells transduced with the uninsulated vector expressed the EGFP transgene to high levels $(E)$, whereas very low level of expression was observed in cells transduced with the sns containing construct $(F)$. In conclusion, results of enhancer blocking assays in two distinct cell lines and with two different vectors suggest that sns maintain directional insulator function in human chromatin.

\section{Analysis of Integrated DNA in Stably}

\section{Transfected Clones}

To ensure that the silencing of EGFP expression in sns-transfected cells was due to a block of enhancer function, the state of the integrated transgene was investigated by Southern blot analyses. DNA was extracted from five independent clones transfected with insulated plasmid vectors (sns-EGFP), displaying almost undetectable green fluorescence, and from one clone transfected with the control plasmid (EGFP) which displayed high levels of fluorescence. Since each transfected plasmid vector has an Xbal recognition site at each end of the tk-EGFP region (see drawing in Fig. 2 for map and probes), digestion of genomic DNA from transfectants with Xbal, followed by hybridization with a CMV-EGFP specific probe should generate a single EGFP hybridization fragment of $875 \mathrm{bp}$ and a single CMV hybridization fragment containing the neo gene and vector sequences, for each integrated trans- 
gene. Detection of these two bands in clone 1 transfected with the uninsulated vector (EGFP) suggests that a single copy plasmid has integrated. Quantitation of the EGFP band intensity by an imaging densitometer suggests that a single intact integrant is also present in the sns containing transfectant clone 4 . In contrast, clones 2 and 6 and clone 5 contain, respectively, two copies and five to six copies of tandem integrants. The size difference between CMV hybridization fragments in clones 2 and 5 can be accounted for the presence in clone 2 of two tandem copies of sns. Finally, the absence of the 875-bp fragment in clone 3 suggests that lack of fluorescence in this particular clone may result from either DNA rearrangement or enhancerpromoter interference. Further insight into the molecular anatomy of integrated DNAs was obtained by hybridization of the same filter, after stripping the CMV-EGFP probe, with a neo probe. The results demonstrated the presence of the neo gene at the same chromosomal site, physically linked to the EGFP reporter sequences. The only exception to this was in clone 5, which appeared to have tandem arrays of plasmids at two different chromosomal sites, in one of which CMV sequences had undergone deletion (Fig. 2A).

Transfected clones that contained the reporter plasmid in an intact form were further analyzed by PCR. Oligonucleotide primers were designed to amplify a DNA segment from the CMV regulative region to a region downstream of the second SV40 polyadenylation signal of the EGFP transcription unit. As shown in Fig. 2B, the sizes of the amplified DNA bands were compatible with the absence of sns in clone 1 and in the plasmid p-EGFP and with the presence of one copy (clones 4-6) or two copies (clone 2) of the sns element. Blot hybridization with EGFP and sns probes (Fig. 2B) confirmed the integrity of the stably integrated transgenes, and the arrangement schematically shown in Fig. 2C. Taken together, these results strongly indicate that the sea urchin sns insulator barred the promoter from the influence of the CMV enhancer when stably integrated in human cells.

\section{Enhancer Blocking Activity at the RNA Leve}

To confirm the enhancer blocking effect, we determined levels of neo and EGFP mRNAs by RNase protection analyses. Total RNA was extracted from transgenic cell lines containing nonrearranged EGFP and EGFP-sns constructs. Assays were performed by using probes for both neo and EGFP in the same hybridization reaction for all, but the uninsulated, transfectants. Results are shown in Fig. 3. Of note, cells stably transfected with the control EGFP plasmid expressed EGFP mRNA at much higher levels than neo mRNA (clone 1), reflecting the higher strength of the CMV enhancer with respect to the SV40 enhancer. In sns constructs,

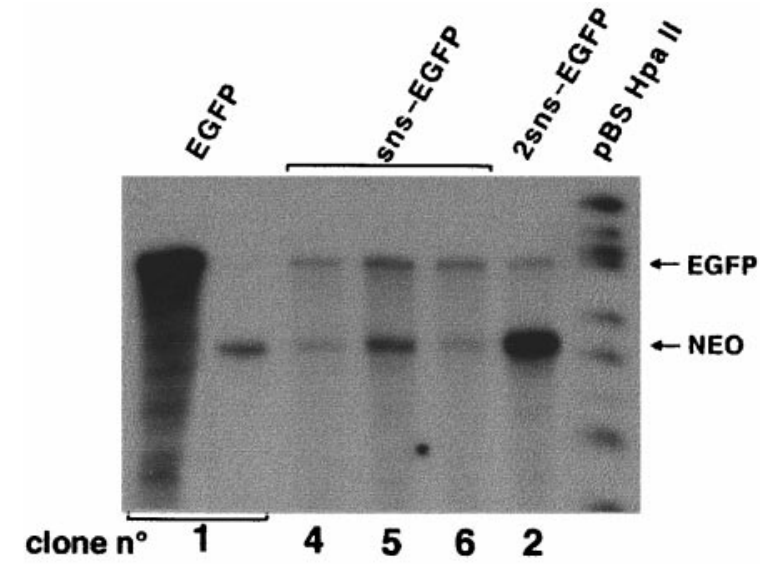

FIG. 3. Transgene expression of transfected cells. RNase protection assay was carried out with $5 \mu \mathrm{g}$ of total RNA extracted from the cellular clones containing nonrearranged integrants. Clone numbers are as in Fig. 2. RNA from clone 1 transfected with the control EGFP, was separately hybridized with antisense EGFP or NEO RNAs. For all other clones the two RNA probes were added to the same hybridization mixture. The protected RNA bands of 345 and 192 bases of respectively EGFP and NEO are indicated.

the level of neo transcripts varied among transfected cell lines. Clone 2 displayed the highest levels of neo mRNA. The higher expression might be due in part to the presence of two copies of the transgene (Fig. 2), and in part to the influence of chromatin on gene expression at the integration site. Inter estingly, expression of the neo transgene in the transfectant containing the tandem arrays (clone 5) was only two or threefold higher than that of the single copy integrant (clones 4 and 6), and much lower than that observed for clone 2. Most importantly, however, is the drastic reduction of EGFP mRNA in all clones transfected with constructs containing sns lying between the CMV enhancer and the promoter. These data confirm that the sea urchin sns insulated the reporter gene from the activating function of the CMV enhancer in human cells.

\section{DNA Binding Activity in Human Cells}

Recently, we have identified three cis-acting sequence elements that are each required for the enhancer blocking function of sns in sea urchin embryos at gastrula stages. These sequence elements are binding sites for specific protein complexes (21). The elements include Box A inverted repeats, Box B direct repeats, and a pyrimidine rich region that contains tandem TC/GA repeats, corresponding to several GAGA sites in the lower strand (see drawing in Fig. 4). Given that sns insulator function is conserved in human cells, we assessed whether the protein binding sites were similarly conserved. We performed an electrophoretic gel mobility shift assay (EMSA) with the three labelled probes, Box A, Box B, and C $+T$ and nuclear extracts from human cells to address this ques- 


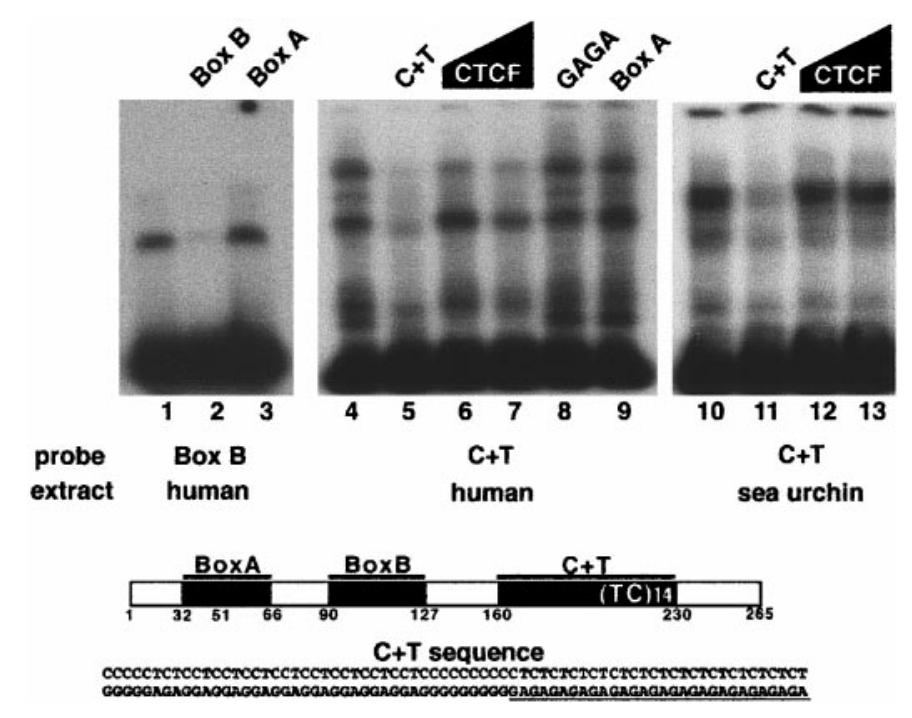

FIG. 4. EMSA analysis of nuclear protein binding sites within sns. Three of the end-labelled probes, Box A, Box B, and C $+T$, are underlined in the schematic drawing of the sns fragment. The sequence of the $\mathrm{C}+\mathrm{T}$ region is shown below the drawing and the GAGA sequence is underlined. The $C+T$ fragment was obtained by PCR amplification. Box A, Box B, CTCF, and GAGA probes were obtained by annealing complementary oligonucleotides. In competition experiments, nuclear extracts were preincubated with $100 \mathrm{ng}$ of unlabelled homologous (lanes 2, 5, and 11), or 100 ng (lanes 6 and 12) or $200 \mathrm{ng}$ (lanes 3, 7-9 and 13) of unlabelled heterologous probes prior the addition of $1 \mathrm{ng}$ of the labelled probe. Lanes 1-9 human nuclear extracts; lanes 10-13, sea urchin nuclear extracts. The DNA-protein complexes were resolved by polyacrylamide gel electrophoresis.

tion. We obtained identical results with nuclear extracts from $\mathrm{H} 1299$ (the cell line in which the enhancer blocking assays were performed) and U937. Box A did not produce detectable specific protein-DNA complexes (not shown), suggesting that this factor is dispensable for the enhancer blocking activity of sns in human cells. This finding is not so surprising given our recent observation in sea urchin. We have in fact not detected Box $A$ binding activity in nuclear extracts from early cleavage embryo (unpublished data), although sns is capable of insulator function at this developmental stage (21). In Fig. 4 are shown the results of the EMSA analysis with the Box $B$ and $C+T$ sites. Both fragments interacted specifically with nuclear proteins as demonstrated by competition assays using excess of unlabelled homologous and heterologous oligonucleotides. Interestingly, while the Box B was assembled in a major DNA-protein complex (lane 1), the $C+T$ fragment interacted with several nuclear proteins (lane 4). We detected two major and a number of less represented complexes that were all competed by 100 fold molar excess of homologous fragment. In sea urchin extracts all of the $\mathrm{C}+\mathrm{T}$ binding activity is due to factors that bind to the CT/AG repeats located at 3 ' end of the pyrimidine tract (lane 10) (21). We addressed the issue of whether a similar human factor binds to sns.
In contrast to the sea urchin extracts, 200-fold molar excess of an oligonucleotide containing the fourteen TC/AG dinucleotides (lane 8) failed to affect the assembly of any of the $\mathrm{C}+\mathrm{T}$ nucleoprotein complexes. In addition, several motives CC(T)CTC required for the binding of CTCF to vertebrate insulator (11), are contained in the pyrimidine tract (see sequence of the $\mathrm{C}+$ $T$ region in Fig. 4). We asked whether this factor binds also to sns. To this end we performed competition mobility shift assays in nuclear extracts from human cells and sea urchin embryos by using a 42-bp oligonucleotide, of the chicken HS4 insulator, containing a single binding site for the CTCF protein (11). The results of Fig. 4 show that of the two major represented complexes only the upper one was competed by 100 and 200 molar excess of the 42 HS4 oligonucleotide (lanes 6 and 7). Conversely, no competition of the sea urchin complexes with excess of CTCF binding site was observed (Fig. 4, lanes 12 and 13). These results would suggest that as for the vertebrate insulators, sns might require the binding of a CTCF related factor for its activity in human cells. Experiments are in progress to address this point.

In conclusion, we have demonstrated the ability of a sea urchin insulator to shield a promoter from the CMV enhancer in two different cell lines using plasmid and retroviral vectors. At least one insulator factor, the Box B binding protein, is probably shared between human and sea urchin. It remains to demonstrate its involvement in the enhancer blocking activity in human cells. The pyrimidine binding proteins seem to be different between the two species. While in sea urchin, a GAGA factor like binds to the CT/GA repeats region (21), in human cells a CTCF related factor and other unknown protein interact with the $\mathrm{C}+\mathrm{T}$ region. Our observations have significant implications for the evoIutionary conservation of mechanisms of insulator action. In addition, the relatively small size and apparent absence of enhancer or species specificity exhibited by sns may prove to be of practical benefit in genetransfer applications.

\section{ACKNOWLEDGMENTS}

Many thanks are due to Dr. Sylvia Evans for reading and editing the manuscript. This work was supported by grants from MURST (Cofinanziamenti Programmi di Ricerca Scientifica di Interesse Nazionale, '99), from FSE (F ondo Sociale Europeo), and from the University of Palermo (ex $60 \%$ ).

\section{REFERENCES}

1. Geyer, P. (1997) The role of insulator elements in defining domains of gene expression. Curr. Opin. Genes. Dev. 7, 242-248.

2. Udvardy, A. (1999) Dividing the empire: Boundary chromatin elements delimit the territory of enhancers. EMBO J . 18, 1-8.

3. Bell, A. C., West, A. G., and Felsenfeld, G. (2001) Insulators and 
boundaries: Versatile regulatory elements in the eukaryotic genome. Science 291, 447-450.

4. Sun, F.-L., and Elgin, S. C. R. (1999) Putting boundaries on silence. Cell 99, 459-462.

5. Kellum, R., and Schedl, P. (1991) A position-effect assay for boundaries of higher order chromosomal domains. Cell 64, 941950.

6. Kellum, R., and Schedl, P. (1992) A group of scs elements function as domain boundaries in an enhancer-blocking assay. Mol. Cell. Biol. 12, 2424-2431.

7. Geyer, P., and Corces, V. G. (1992) DNA position-specific repression of transcription by a Drosophila zinc finger protein. Genes and Dev. 6, 1865-1873.

8. Cai, N. H., and Levine, M. (1995) Modulation of enhancerpromoter interactions by insulators in the Drosophila embryo. Nature 376, 533-536.

9. Scott, S. K., and Geyer, P. K. (1995) Effects of the su(H w) insulator protein on the expression of the divergently transcribed Drosophila yolk protein genes. EMBO J . 24, 6258- 6257.

10. Chung, J. H., Whiteley, M., and Felsenfeld, G. (1993) A 5 ' element of the chicken $\beta$-globin domain serves as an insulator in human erythroid cells and protects against position effects in Drosophila. Cell 74, 505-514.

11. Bell, A. C., West, A. G., and Felsenfeld, G. (1999) The protein CTCF is required for the enhancer blocking activity of vertebrate insulators. Cell 98, 387-396.

12. Recillas-Targa, F., Bell, A. C., and Felsenfeld, G. (1999) Positional enhancer-blocking activity of the chicken $\beta$-globin insulator in transiently transfected cells. Proc. Natl. Acad. Sci. USA 96, 14354-14359.

13. Bell, A. C., and Felsenfeld, G. (2000) Methylation of a CTCFdependent boundary controls imprinted expression of the Igf2 gene. Nature 405, 482- 485.

14. Hark, A. T., Schoenherr, C. J., Katz, D. J ., Ingram, R. S., Levorse, J. M., and Tilghman, S. M. (2000) CTCF mediates methylation-sensitive enhancer-blocking activity at the $\mathrm{H}$ 19/l gf2 locus. Nature 405, 486- 489.

15. Pikaart, M. J ., Recillas-Targa, F., and Felsenfeld, G. (1998) Loss of transcriptional activity of a transgene is accompanied by DNA methylation and histone deacetylation and is prevented by insulators. Genes and Dev. 12, 2852-2862.

16. Wang, Y., F. J ., DeMayo, S., Tsai, and O'Malley, B. W. (1997) Ligand-inducible and liver-specific target gene expression in transgenic mice. Nat. Biotechnol. 15, 239-243.

17. Emery, D. W., Yannaki, E., Tubb, J ., and Stamatoyannopoulos, G. (2000) A chromatin insulator protects retrovirus vectors from chromosomal position effects. Proc. Natl. Acad. Sci. USA 97, 9150-9155.

18. Rivella, S., Callegari, J . A., May, C., Tan, C. W., and Sadelain, M. (2000) The cHS4 insulator increases the probability of retroviral expression at random chromosomal integration sites. J . Virol. 74, 4679-4687.

19. Steinwaerder, D. S., and Lieber, A. (2000) Insulation from viral transcriptional regulatory elements improves inducible transgene expression from adenovirus vectors in vitro and in vivo. Gene Ther. 7, 556-567.

20. Palla, F., Melfi, R., Anello, L., Di Bernardo, M., and Spinelli, G. (1997) Enhancer blocking activity located near the $3^{\prime}$ end of the sea urchin early H2A histone gene. Proc. Natl. Acad. Sci. USA 94, 2272-2277.

21. Melfi, R., Palla, F., Di Simone, P., Alessandro, C., Calì, L., Anello, L., and Spinelli, G. (2000) Functional characterization of the enhancer blocking element of the sea urchin early histone gene cluster reveals insulator properties and three essential cis-acting sequences. J . Mol. Biol. 304, 753-763.

22. Palla, F., Bonura, C., Anello, L., Di Gaetano, L., and Spinelli, G. (1994) Modulator factor-binding sequence of the sea urchin early histone H2A promoter acts as an enhancer element. Proc. Natl. Acad. Sci. USA 91, 12322-12326. 